ISSN: 0213-2060

DOI: https://doi.org/10.14201/shhme2020381728

\title{
GENTES DEL ISLAM. RITUAL FUNERARIO E HISTORIAS DE VIDA EN LA MAQBARA DE PAMPLONA
}

\author{
People of Islam. Funerary Ritual and Life Stories in the maqbara of Pamplona
}

\author{
M. a Paz de MIGUEL IBÁÑEZ \\ Depto. de Prehistoria, Arqueología, Historia Antigua, Filologia Griega y Filologia Latina. Facultad de Filosofía y \\ Letras II. Universidad de Alicante. Ctra. San Vicente del Raspeig, s/n. E-03690 SAN VICENTE DEL RASPEIG \\ (Alicante).C.e.:pdm@ua.es
}

Recibido: 2019-11-28

Revisado: 2020-03-24

Aceptado: 2020-05-04

RESUMEN: Presentamos algunos resultados obtenidos del estudio osteoarqueológico realizado sobre los restos exhumados en la maqbara de la Plaza del Castillo de Pamplona. De las 172 sepulturas islámicas identificadas se han recuperado 177 esqueletos en diferentes estados de conservación. Demográficamente representa una población natural, propia de sociedades prevacunales, donde casi la mitad de la población falleció antes de alcanzar la edad adulta. Los datos patológicos han permitido reconocer diferentes enfermedades de origen infeccioso, metabólico, congénito y tumoral, entre otros. Destacan las lesiones de origen violento, que en cinco casos produjeron la muerte de los individuos, todos ellos hombres. Genéticamente se reconoce una población compleja con marcadores africanos, locales y mixtos. La identificación de manipulaciones dentales intencionadas, preferentemente en mujeres, permite reconocer marcadores culturales originariamente africanos, presentes igualmente en población genéticamente local, signo claro de la aculturación de parte de la población autóctona.

Palabras clave: Islamización; Demografía; Paleopatología; Violencia; Aculturación.

ABSTRACT: We present some results obtained from the osteoarcheological study carried out on the remains exhumed in the maqbara of the Plaza del Castillo de Pamplona. Of the 172 Islamic graves identified, 177 skeletons have been recovered in different conservation states. Demographically, it represents a natural population, typical of pre vaccine societies, where almost half of the population died before reaching adulthood. The pathological data have allowed recognizing different diseases of infectious, metabolic, congenital and tumor origin, among others. Injuries of violent origin are highlights, which in five cases resulted in the death of individuals, men in all cases. A complex population with 
African, local and mixed markers is genetically recognized. The identification of intentional dental manipulations, preferably in women, allows us to recognize originally African cultural markers, also present in the genetically local population, a clear sign of acculturation on the part of the native population.

Keywords: Islamization; Demography; Paleopathology; Violence; Acculturation.

SUMARIO: 0 Introducción. 1 La nueva mirada. 2 La excavación y sus inesperados hallazgos. $3 \mathrm{La}$ información contenida en las tumbas. Osteoarqueología. 4 De la Osteoarqueología al reconocimiento de la migración y la aculturación. 5 Lo que nos cuenta la población islámica de Bambeluna (Pamplona). 6 A modo de conclusión. 7 Referencias bibliográficas.

\section{INTRODUCCIÓN}

Los estudios realizados en la maqbara de Pamplona ofrecen una información relevante para el conocimiento histórico, tanto del norte como del resto del territorio peninsular. Pasado un tiempo desde la realización de la tesis doctoral accedemos a la información obtenida con una nueva mirada, rescatando los datos conocidos sobre demografía, paleopatología y rasgos culturales que permiten mejorar su interpretación.

El cementerio islámico fue excavado en 2002 documentando un ritual claramente islámico con una población representativa de una comunidad demográficamente natural. $\mathrm{Su}$ estudio paleopatológico ofrece un amplio abanico de estados de salud, con algunos casos en los que se ha podido determinar la causa de muerte, preferentemente a partir de las heridas visibles en los esqueletos.

Los marcadores genéticos, isotópicos y culturales han permitido reconocer una comunidad por el momento única en la que confluyen gentes alóctonas, procedentes del norte de África, y autóctonas.

Es relevante destacar, igualmente, que Pamplona conservó su identidad cristiana, hecho documentado en las dos necrópolis con cronologías entre los siglos viI y viII, habiendo convivido directamente con la población islámica asentada en la ciudad.

\section{LA NUEVA MIRADA}

La evidencia de estar ante una población que utilizó el ritual islámico tras la muerte nos planteó, entre otros objetivos, los que queremos recoger en este trabajo. Por una parte, el reconocimiento de la presencia de nuevas gentes, hasta hace pocos años casi invisibles, presentes en los inicios de la islamización en Pamplona. Una población que se ha podido reconocer a través de su rito funerario, de indudable vínculo con el Islam, hecho que inicialmente creó un profundo desconcierto entre quienes investigaban la Alta Edad Media en Navarra y en el resto del territorio peninsular. Las fuentes escritas apenas 
permitían sospechar esta presencia, ya que las menciones a la llegada de gentes del islam a Pamplona fueron generalmente consideradas de poca fiabilidad.

Una nueva perspectiva se sumó a las investigaciones realizadas hasta el momento del hallazgo del cementerio, la que ofrece el estudio osteoarqueológico, ofreciendo datos sobre la demografía, uno de los campos que permite conocer las características de la población exhumada.

El reconocimiento de la ritualidad islámica documentada sobre el terreno nos planteó varias opciones sobre el origen poblacional. Por una parte, que fueran personas procedentes de tierras lejanas, quizás africanas, que se asentaron, vivieron y murieron en Pamplona. De este modo surgió la duda de si pudiéramos estar ante un grupo de población local que, a partir de los influjos del nuevo poder político, se convirtieron a la nueva religión. A estas dos posibilidades se ańade una tercera, la de estar ante un grupo mixto, en el que se encontraran gentes del islam y personas locales que, por motivos que de momento nos son desconocidos, decidieran asumir unas creencias que en principio les eran ajenas, asociando sus vidas a las del grupo recién llegado. En este campo dos estudios han permitido obtener interesantes resultados, el principal, la tesis doctoral de Lara Fontecha (2013), y la nuestra en la que se reconocen marcadores dentales de carácter cultural (Romero et ál., 2010; De Miguel, 2016) de gran relevancia para elaborar nuestras conclusiones.

En definitiva, el principal objetivo es presentar, a partir de los datos obtenidos en los estudios, la población islámica de Pamplona como una sociedad compleja, en la que tanto hombres como mujeres tuvieron un protagonismo del que sus restos funerarios son el fundamento de la información.

\section{LA EXCAVACIÓN Y SUS INESPERADOS HALLAZGOS}

El hallazgo de una maqbara en el centro neurálgico de la ciudad de Pamplona, en los albores del siglo Xxi (2002), fruto de un trabajo arqueológico vinculado a la obra que construiría un aparcamiento en la Plaza del Castillo, supuso el replanteamiento de los inicios de la Conquista islámica, tanto a nivel local como peninsular. Esta actuación requirió la excavación y documentación de un amplio abanico de niveles históricos que, no por ser esperados -basta con el topónimo-, en algunos momentos resultaron ser absolutamente novedosos. Así ocurrió con un lienzo de muralla de cronología probablemente bajomedieval y unas termas romanas, si bien dentro de lo absolutamente imprevisto estaba la maqbara, cuyas evidencias rituales (fosa simple, enterramiento individual, decúbito lateral derecho, mirando al Este-Sureste) no permitían ninguna duda sobre su filiación.

La localización del cementerio islámico planteaba, como hemos indicado, un reto histórico importante que pasaba por conocer quiénes eran esas personas, su número, sus edades y sexos, las características físicas, enfermedades y, si fuera posible, su origen genético. De esas preguntas surgió la posibilidad de realizar dos trabajos que, pasado 
el tiempo, se convirtieron en dos tesis doctorales: una centrada en la Osteoarqueología integrando los aspectos históricos de una época aun velada (De Miguel, 2016), la segunda centrada en la genética poblacional (Fontecha, 2013). Con estos estudios buscamos obtener unos resultados científicamente válidos. Hay que recordar la gran complejidad técnica en los procedimientos que permiten recuperar suficiente información genética de los restos humanos arqueológicos. A pesar de estos dos trabajos, y de una tercera tesis sobre la Arqueología de Pamplona (García-Barberena, 2016), fundamentales para el conocimiento de esta población, entre la ciudadanía aún perdura la opinión de que nada se hizo con los restos recuperados, considerando a nivel popular que de nada sirvió un trabajo arqueológico que tan interesantes resultados científicos han dado.

Pasado un tiempo de reflexión tras las tesis y habiendo recibido las consideraciones de diferentes colegas de elevada relevancia científica, consideramos que el trabajo realizado, a pesar de no ser definitivo, ofrece una nueva mirada sobre unos momentos históricos que han permanecido en tinieblas y que esta excavación comienza sutilmente a desvelar.

\section{La INFORMACión CONTENIDA EN LAS TUMBas. OsteOARQUEOLOGÍA}

Toda investigación comienza en un momento más o menos determinado, aunque rara vez finaliza por completo. Este es el caso de esta población islámica sobre la que aún tenemos proyectos abiertos que esperamos poder compartir en un tiempo razonable.

La Osteoarqueología permite obtener a partir del estudio de los restos humanos esqueletizados, procedentes de yacimientos arqueológicos, datos relacionados con el número mínimo de individuos, sus edades, sexos, características físicas (talla, índices de robustez y signos de actividad, entre otros), paleopatología y, como ocurre en nuestro caso, modificaciones corporales de origen cultural. La documentación se inicia en el momento de la excavación, partiendo de un buen registro en el campo (Duday et ál., 1990), recogiendo todos los datos que permitan conocer tanto el gesto funerario, con clara intencionalidad en el momento de la inhumación, como todos los procesos tafonómicos ocurridos tras el depósito del cuerpo en la sepultura.

Tras su exhumación y cuidadoso transporte al laboratorio se procede a su limpieza y reconstrucción, en la medida en que su estado de preservación lo requiera. Los materiales óseos son inventariados, medidos y fotografiados, creando un registro de datos básico para el conocimiento de las características físicas y de conservación de cada esqueleto.

La determinación del número mínimo de individuos junto con las edades y los sexos nos permite ofrecer información sobre la paleodemografía a partir de las propuestas de Bocquet-Appel (2008) y del índice de juventud (IJ) utilizado para el reconocimiento de sociedades en estado de crecimiento o decrecimiento poblacional.

Una vez preparados los esqueletos intentamos recuperar datos relacionados con la edad, diferenciando entre individuos infantiles y adultos. Durante la infancia la determinación de la edad se basa preferentemente en la fase de desarrollo dental, lo que permite 
precisarla con un ligero margen de error (Ubelaker, 2007: 84). En el caso de perinatales son las medidas óseas las que nos permiten proponer una edad, en ocasiones menor a las 40 semanas de gestación, correspondientes con el desarrollo fetal normal en nuestra especie. Estas dimensiones se aplican igualmente en los casos de gestantes identificadas en las excavaciones o en el laboratorio, como ha ocurrido en Pamplona (Balthazard y Dervieux, 1921; Fazekas y Kósa, 1978; Jeanty et ál., 1984; Scheuer y Black, 2000). En las personas adultas los criterios son menos precisos, por lo que se han utilizado varios con el fin de evitar, en la medida de lo posible, los errores (Buikstra y Ubelaker, 1994; Ubelaker, 2007); a pesar de ello hemos considerado adecuado agruparlos de forma amplia -adulta joven, adulta, adulta madura y senil-.

En la identificación del sexo partimos de la premisa de que morfológicamente es muy complicado su determinación en población infantil, a pesar de las propuestas realizadas por algunos autores para su determinación en los esqueletos que no han llegado a la adolescencia, momento del desarrollo de los caracteres sexuales secundarios (Schutkowski, 1993). Es en esa fase de desarrollo cuando se van definiendo las características sexuales esqueléticas, preferentemente en la pelvis, como es bien conocido. El trabajo de Ferembach y colaboradores (1979) ha sido la base principal del cotejo, recogido en numerosas publicaciones de referencia posteriores como el caso de Buikstra y Ubelaker (1994), para la identificación sexual en población juvenil y adulta.

Antropométricamente hemos utilizado las medidas necesarias para hacer una clasificación de las tipologías craneales en dolicocráneo, mesocráneo y braquicráneo. De igual modo, se han obtenido las dimensiones de los huesos largos para aproximarnos a las tallas según diferentes propuestas: Nunes de Mendonça (1998), Pearson (1899), y Trotter (en Ubelaker, 2007: 81).

Otro aspecto recogido es el reconocimiento de los estados de salud, previos obviamente al fallecimiento. Hemos intentado, a partir de las alteraciones óseas y dentales, conocer si la persona padeció episodios de enfermedad y si fueron superados o se convirtieron en la causa que provocó su muerte.

La genética se hace fundamental en nuestra población dado el interés por resolver cuestiones clave relacionadas con su origen local o alóctono. La tesis doctoral de Lara Fontecha defendida en 2013 ha permitido esclarecer datos sumamente relevantes al respecto.

En relación con los isótopos de movilidad, tan solo tenemos datos de dos mujeres con unos resultados de gran interés para proponer el posible origen de parte de la población (Prevedorou et ál., 2010).

Un aspecto que resultó novedoso es el reconocimiento de modificaciones dentales de carácter intencional. Estas denticiones se han estudiado con microscopía electrónica de barrido (EMB), en un intento por diferenciar los desgastes de origen alimentario de las posibles, aunque infrecuentes, manipulaciones dentarias de origen cultural (Romero et ál., 2009). 
Para poder valorar justamente los datos obtenidos de la población de Pamplona, en primer lugar hay que considerar que la maqbara no está excavada en su totalidad, por lo que los resultados presentados siempre serán parciales, respecto a la población realmente inhumada en la necrópolis.

Del total de tumbas identificadas como islámicas -172- se recuperaron restos de 177 individuos. Las sepulturas eran individuales con el matiz de un área, donde se concentraron cinco perinatales, en la que no se pudo diferenciar si se trataba de una sepultura múltiple o de varias individuales concentradas en un reducido espacio. Dos tumbas de mujeres fallecidas durante la gestación deberían ser consideradas como individuales, dado que en el momento del entierro solo se depositó un cuerpo, aunque en el momento de la exhumación se identificaron dos individuos, la madre y los restos fetales.

El ritual funerario identificado fue claramente islámico: enterramientos individuales, en decúbito lateral derecho, en ocasiones con desplazamientos debidos a los procesos tafonómicos, orientados de forma que la mirada quedara hacia el Este-Sureste. Carecen de elementos de ajuar u objetos acompañantes relacionados con las vestimentas, adornos $\mathrm{u}$ ofrendas. Esta norma se ve truncada en tres casos; en uno se conservaba un pequeño anillo asociado a un esqueleto adulto de sexo indeterminado, una placa de bronce a la altura del hombro izquierdo junto a una mujer fallecida durante el embarazo, y un hombre juvenil con un fragmento de cuerno cortado con instrumento de filo.

Por la posición de los esqueletos, la mayoría de las veces conservados en conexión anatómica, con pequeños desplazamientos, consideramos que lo habitual fue el entierro de los cuerpos amortajados con telas, sin elementos metálicos de anclaje o cierre. La colmatación de la tumba debió de ser temprana en la mayoría de los casos, si bien el desplazamiento del cuerpo completo a decúbito prono o supino en varias sepulturas, indica que tras su depósito el espacio estaba vacío, lo que permitió estos movimientos en el interior de la tumba. Durante la excavación se identificaron algunos cuerpos calzados con piedras colocadas a su espalda, en un intento de conservar la posición canónica en decúbito lateral, evitando su desplazamiento. En un reducido número de sepulturas se identificaron cubiertas de lajas, generalmente a un agua. Un caso singular fue la sepultura 54, una cista formada de mampuesto, perteneciente a un individuo infantil de 3-4 años, en la que el tamaño del esqueleto era considerablemente menor que el espacio de la sepultura, por lo que consideramos que se trata de la utilización de una sepultura preparada para una persona y que fue aprovechada para otra a la que inicialmente no estaba destinada (Figura 1).

En el espacio destinado a las sepulturas de rito islámico se han identificado diez que por sus características se encuadran dentro del rito cristiano. Se sitúan de forma dispersa, con la excepción de tres que aparecen agrupadas en el área suroeste de la plaza, elaboradas con lajas o mampuesto y con los cuerpos en decúbito supino, mirando hacia el este, en algunos casos con ajuar acompañante (De Miguel, 2016: 164, 167). Esta representación cristiana en un área claramente islámica, no es excepcional; se ha documentado en otros ámbitos peninsulares como Jaén (Serrano y Castillo, 2000), Zaragoza (Galve, 1995: 


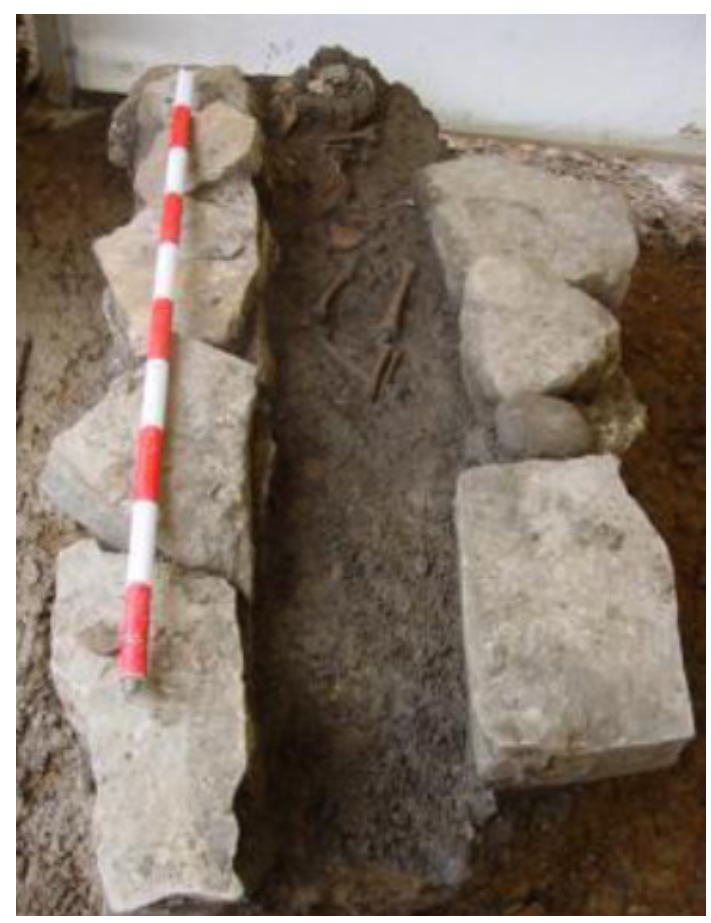

Figura 1. Sepultura 54, estructura incompleta de mampostería. Infantil 3-4 ańos, decúbito lateral derecho desplazado por tafonomía (Fotografía: Trama S. L.).

118), El Tossal de Manises (Alicante) (Tendero et ál., 2007: 184), el Tolmo de Minateda (Hellín, Albacete) (Gutiérrez, 2013: 243), Segóbriga (Almagro Bach, 1975) y Madrid (Vigil-Escalera, 2009), barajándose la hipótesis de una continuidad poblacional a pesar del cambio de ritual y, por tanto, de creencias religiosas en el tránsito del siglo viII al Ix. En nuestro caso podemos proponer tres hipótesis: la primera, que se trate de una utilización previa del espacio como área cementerial de población cristiana; la segunda, que se enterrara una pequeña parte de población cristiana junto con la islámica, por motivos de parentesco o afinidad, poco probable a nuestro entender; y la tercera, que el espacio se utilizara de forma puntual, quizás por población islámica convertida al cristianismo tras desplazar el poder islámico por el cristiano en los albores del siglo ix. En un intento de disponer de más elementos de juicio se realizó la datación de los restos procedentes de la sepultura 97, un individuo infantil que estaba acompañado por un anillo de gran calidad, si bien el margen cronológico ofrecido por la muestra, entre los siglos VII-IX, no nos permite aclarar este asunto (Beta 248602. 1240 440 BP; 670-890 AC, 2 sigmas; intercept of radiocarbon age with calibration curve, $770 \mathrm{cal} \mathrm{AD} \mathrm{(cal} \mathrm{BP} \mathrm{1180))} \mathrm{(De} \mathrm{Miguel,}$ 2016: 132). 
A partir de la identificación de las sepulturas con ritual islámico, de las que tan solo el propio ritual nos confirma su pertenencia a la nueva religión arribada con la conquista, y de que carecemos de materiales asociados, sin más elementos de juicio que los esqueletos, son los datos obtenidos de su estudio la base fundamental para comprender este velado momento histórico. Para precisar la cronología se realizaron dos dataciones por AMS, coincidiendo ambas en el siglo viII (sepultura 32, Beta 218654; Cal AD 660-770; sepultura 159, Beta 248601; Cal AD 650-770 AC), sabiendo que no pueden ser anteriores a 712-718, momento en el que se firmó el Pacto de Pamplona.

Los datos obtenidos nos muestran una población demográficamente normal, que corresponde con una población estable, con una alta presencia infantil y subadulta. Hay tanto mujeres como hombres, si bien estos son algo más numerosos. El abanico de edades abarca desde el periodo fetal, el nacimiento, con varios perinatales, hasta la senectud, aunque solo los hombres están representados en edades superiores a los 55-60 años (Gráfico 1).

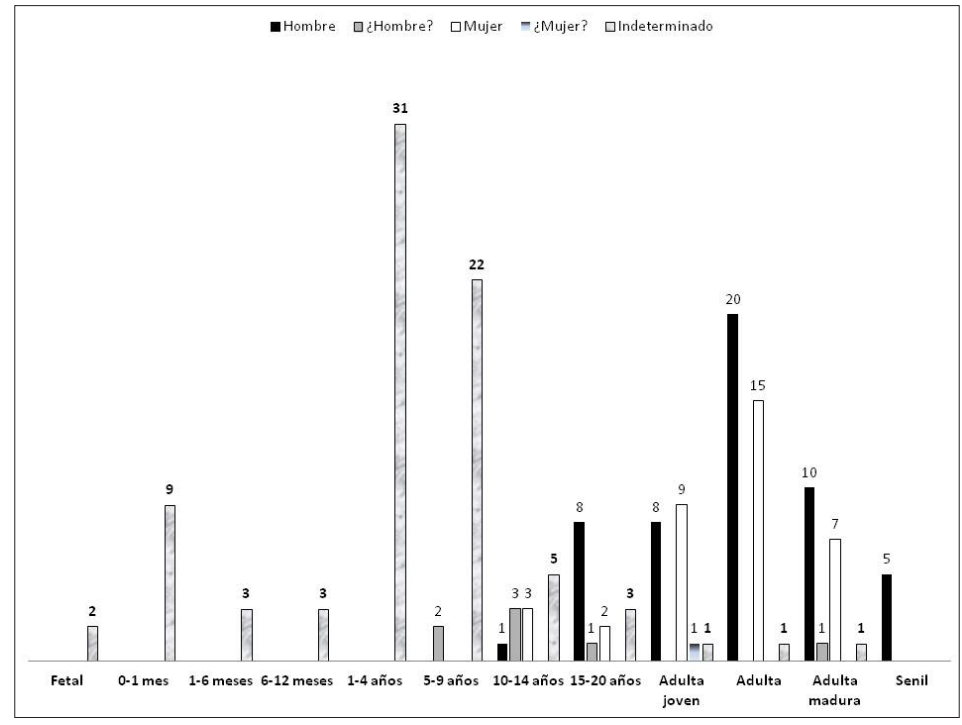

Gráfico 1. Perfil demográfico de la maqbara de Pamplona.

De los datos aportados sobre la población infantil, entendida esta como aquella que no ha alcanzado la pubertad, esto es, la menor de 14 ańos, poniendo esta edad biológica de modo convencional, conscientes de posibles errores difíciles de subsanar (Gráfico 2), destaca en este grupo la baja representación de perinatales (entre el nacimiento y los 28 días de vida) y lactantes, considerados estos en una edad entre el primer mes de vida y los dos años. Son un total de diez lactantes, seis fallecieron durante el primer año y cuatro en el segundo. Los seis primeros llevarían una vida absolutamente dependiente, preferentemente de los cuidados de su madre, y circunstancialmente de otras personas 
del grupo familiar. En el segundo año de vida, es normal el inicio de la deambulación y del consumo de nuevos alimentos, comenzando el cambio de la alimentación materna a la del resto de la familia. Es el momento en el que comienzan los riesgos de accidentes, y de la ingesta de alimentos con riesgo de contaminación lo que pudo provocar infecciones gastrointestinales y parasitarias. Es el grupo de edad entre los 3-4'9 ańos, el más numeroso entre la población no adulta. Se identificaron 28 enterramientos, siendo complejo determinar una sola causa para este número tan elevado; parece que el cese de la lactancia puede relacionarse con esta elevación de la mortalidad, recogiéndose en el Corán de forma explícita recomendaciones al respecto: «Las madres amamantarán a sus hijos durante dos años completos si desea que la lactancia sea completa. [...] Y no hay inconveniente en que el padre y la madre quieran, de mutuo acuerdo y luego de consultarse, destetar al niño. Y, si queréis emplear a una nodriza para vuestros hijos, no hacéis mal, siempre que paguéis lo acordado conforme al uso» (Corán, 2, 233). Es, por tanto, aceptable considerar que las personas creyentes, conocedoras del Corán, mantendrían de forma bastante generalizada la lactancia materna durante los dos primeros ańos de vida, creando un vínculo de dependencia entre la hija/o con la madre, lo que probablemente limitaría su libertad de actuación. Eso no implica que hacia el final del primer ańo se inicie el consumo de nuevos alimentos, hecho propio de cualquier grupo que tenga situaciones de socialización como las comidas en grupos familiares más o menos extensos. Cuando se empieza a caminar, hecho que ocurre de forma generalizada durante el segundo ańo de vida, accederá a fuentes de alimentación disponibles para el resto de la familia y, por tanto, se iniciará en la práctica social de la alimentación.

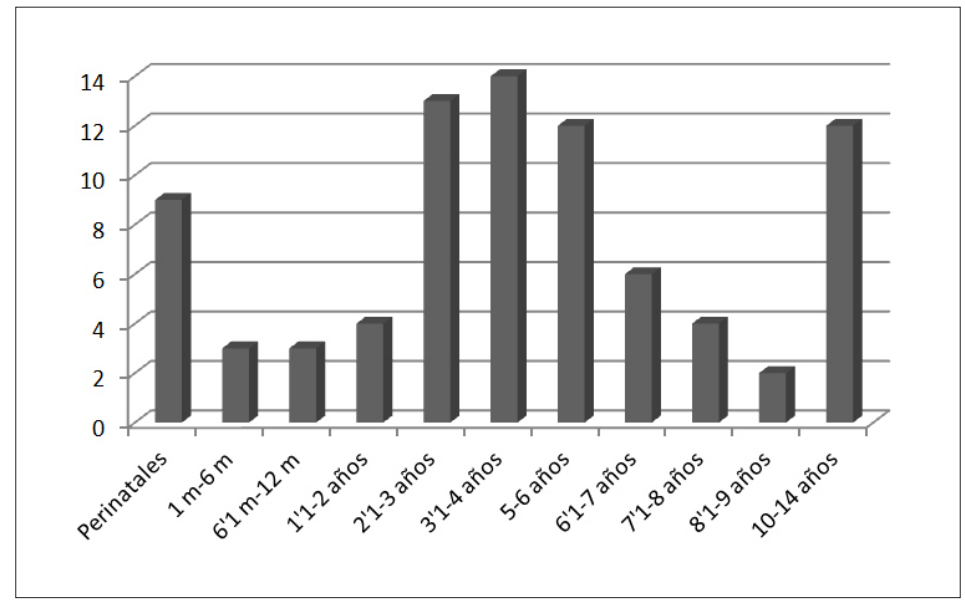

Gráfico 2. Distribución de la población infantil y juvenil.

Si observamos, en la maqbara de Pamplona hay un elevado número de fallecimientos entre los 2'1-3 años con 13 representaciones, y entre los 3'1-4 años con 14. Sin descartar el papel beneficioso de la lactancia prolongada más allá de los dos años, culturalmente 
establecido en esta comunidad de creyentes como hemos sugerido, el papel beneficioso de la lactancia materna como fuente de calorías, así como la acción protectora frente a patologías infecciosas va disminuyendo a lo largo de la misma, a la vez que aumentando la exposición de los infantiles a infecciones, accidentes y a las enfermedades exantemáticas que debieron tener un importante impacto en la morbi-mortalidad durante los primeros años de vida. De hecho, hemos identificado dos casos de sinusitis activa, en la sepultura 43 (3 años) y en la sepultura 73 (3-4 años), que parecen indicar que pudieron fallecer por complicaciones respiratorias de origen infeccioso.

La vida sigue su curso y la presencia de diferentes grupos de edad en la maqbara se documenta con claridad. Son relativamente elevadas las muertes entre los 5-9 años -24 casos-, fase de la vida en la que se va adquiriendo mayor independencia y responsabilidades colaborativas dentro del grupo, preferentemente de la familia, como la ayuda con el ganado, en el trabajo del campo, en el cuidado de menores y en el apoyo de actividades cotidianas de mantenimiento (acarreo de agua, recogida de combustible). No conocemos con claridad las causas de muerte a esas edades, si bien las patologías infecciosas, de nuevo, debieron tener gran responsabilidad en las mismas. De hecho, el infantil de la sepultura 20 (6-7 ańos) muestra signos costales que bien pudieran corresponder con tuberculosis.

Los signos patológicos más abundantes en población infantil desde lactantes a la pubertad son la criba femoral con 44 casos y la criba orbitaria con 40 casos identificados. Estas alteraciones se relacionan con episodios de anemia cuyas causas pueden ser muy diversas (metabólicas, infecciones, parásitos).

Una excepción demográfica se observa en la representación de la población juvenil (15-20 ańos) en la que reconocemos preferentemente hombres (hombres: 8; ¿hombre?: 1; mujeres: 2; indeterminado: 3). Es habitual que sean las mujeres las más numerosas en esta etapa vital, al coincidir con el inicio de la fase reproductiva, en ocasiones de forma precoz durante la adolescencia, por lo que las probables complicaciones vinculadas a los embarazos tempranos, y los partos en cuerpos aun no talmente desarrollados, hagan que el riesgo vital ante estas nuevas situaciones se incremente en mujeres tan jóvenes. En el caso de los hombres este periodo suele ser de menor mortalidad en condiciones naturales. En nuestro estudio la anomalía que presenta el hecho de que haya considerablemente más hombres que mujeres en este rango de edad ( $9 / 2$, más 3 indeterminados), puede justificarse ante la idea de que los hombres de este grupo tienen funciones militares que expondrían a los jóvenes a riesgos vitales relacionados con enfrentamientos interpersonales o bélicos, hecho constatado a partir de signos de violencia en dos de los esqueletos.

La población adulta representa aproximadamente el $50 \%$ de los restos recuperados. Se observa un cierto predominio de hombres respecto de mujeres, siendo entre los 30-45 años donde hay mayor representación, de ambos sexos. Un dato a considerar es que tan solo cinco individuos superaron los 55-60 ańos de vida, todos ellos hombres, hecho para el que por el momento no tenemos clara su justificación.

Las características antropológicas nos presentan una población preferentemente de tipología craneal mediterránea, aunque no de forma exclusiva. Los hombres tienen una 
talla media de $167^{\prime} 17 \mathrm{~cm}$, y las mujeres de $156^{\prime} 6 \mathrm{~cm}$, muy similares a otras poblaciones medievales peninsulares (Tabla 1).

\begin{tabular}{|l|c|c|}
\hline Necrópolis & Hombres & Mujeres \\
\hline Palat del Rey (León) & $163^{\prime} 83$ & $154^{\prime} 57$ \\
\hline Marialba & $171^{\prime} 87$ & $154^{\prime} 50$ \\
\hline La Olmeda & $166^{\prime} 82$ & $160^{\prime} 67$ \\
\hline Vascos medievales & $167^{\prime} 40$ & $157^{\prime} 80$ \\
\hline Tossal de Manises (Alicante) & $168^{\prime} 07$ & $158^{\prime} 81$ \\
\hline Raval (Crevillent, Alicante) & $167^{\prime} 10$ & $153^{\prime} 60$ \\
\hline Maqbara de Pamplona & $167^{\prime} 17$ & $156^{\prime} 60$ \\
\hline
\end{tabular}

TABLA 1. Relación de tallas por sexos en centímetros, según Trotter y Gleser (Prada, 1993: 266, tabla 248; Roca de Togores, 2008: 68; De Miguel, 2009) (De Miguel, 2016: 650, tabla 6.4.).

Los aspectos relacionados con las enfermedades y causas de muerte entre la población adulta son complejos. Parece claro sugerir que la mayoría de los casos se vinculan con complicaciones infecciosas, que de forma habitual no dejan huellas óseas. No obstante, tenemos un caso identificado con lepra, correspondiente con un hombre adulto joven (sepultura 162); y dos casos de tuberculosis en hombres (sepulturas 13 y 112), en una mujer (sepultura 28) y en un indeterminado (sepultura 37). Casos que se unen a los no adultos con lesiones costales de probable origen tuberculoso (sepulturas 20, $91 \mathrm{y}$ 185). Todas estas infecciones requieren un tiempo de desarrollo de la enfermedad para que sus signos queden sobre los huesos, no causando una muerte rápida.

Entre las causas probables de muerte hay dos que han quedado bien diferenciadas entre los sexos. La primera, propiamente de mujeres, que parecen relacionarse con complicaciones surgidas durante el embarazo o el parto (sepulturas 119 y 140), documentándose durante la excavación la aparición de restos esqueléticos de las dos mujeres con restos fetales situados preferentemente en su cavidad pélvica. A pesar de estas evidencias, no podemos descartar que la causa de muerte fuera ajena al embarazo, como pueden ser accidentes o infecciones que pudieron verse agravadas por la gestación.

Los traumatismos y signos violentos no se documentan hasta la juventud (mayores de 15 ańos). Destacan, por su representación, las lesiones claramente vinculadas con enfrentamientos violentos. No hay evidencias de muertes en batallas, más bien parecen casos de enfrentamientos interpersonales, no descartando en alguno de ellos las muertes por ajusticiamiento, como el individuo 32, donde se observa la decapitación a partir de dos cortes muy violentos, uno claramente en el cráneo y el segundo que afecta a las apófisis mastoides, a las dos primeras vértebras cervicales (atlas y axis) y a las ramas ascendentes mandibulares (Figura 2). 


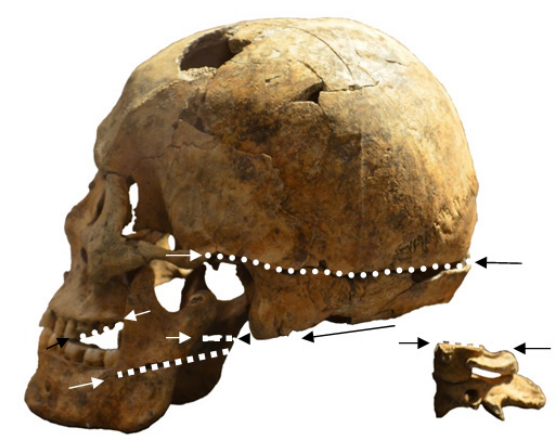

Figura 2. Cráneo con signos de decapitación. Dos golpes de espada. Sepultura 34.

En otros casos, como en las sepulturas 61 y 92, encontramos varios golpes de espada o hacha, en el primero seis y en el segundo 18 , lo que parece indicar un acorralamiento con ensañamiento durante el enfrentamiento, quizás contra más de un individuo simultáneamente (Figura 3).
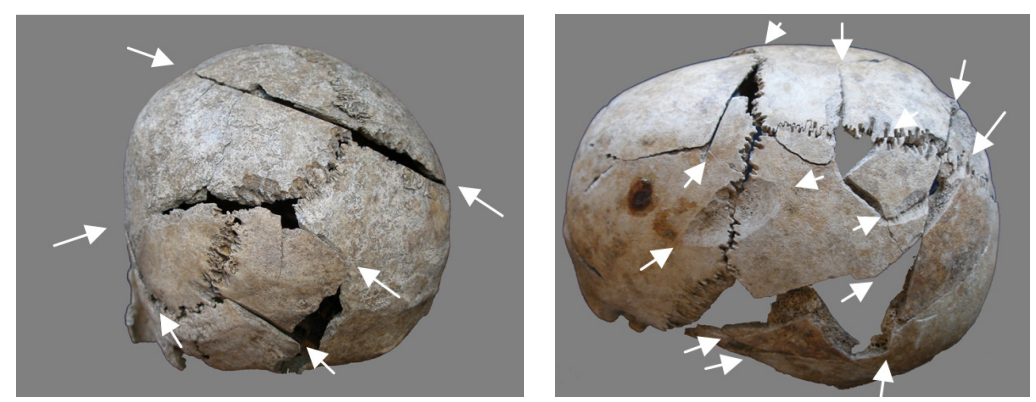

FiguRa 3. Lesiones por arma blanca, sepulturas 61 y 92.

Además de estas muertes violentas hay que destacar otro caso con lesión craneal por arma blanca, en el que se constata una larga supervivencia (sepultura 78). Del mismo modo debemos reseñar otras evidencias traumáticas de las que descartamos su origen accidental. En diez de los enterramientos se documenta fractura de cúbito o de cúbito y radio, claramente cicatrizadas. El origen de estas lesiones, denominadas fracturas de Parry o de "parada de golpe» es una agresión violenta con un objeto contundente que es frenado con el antebrazo lesionando únicamente el cúbito, o el cúbito y el radio. Esta circunstancia es poco frecuente entre otras poblaciones medievales navarras actualmente en estudio (necrópolis de la casa del Condestable -Pamplona- y maqbara de la calle Herrerías -Tudela-), aunque bien identificada en algunos contextos prehistóricos con marcadas connotaciones de violencia en su población, como San Juan Ante Portam Latinam (La Guardia, Álava) (Etxeberria et ál., 1996), donde se documenta de forma más 
numerosa entre los hombres, siendo considerado un indicador de la existencia de violencia interpersonal en las comunidades estudiadas.

Otro grupo de personas que nos resultó interesante analizar genéticamente, cuando iniciamos el estudio, fue el de quienes mostraban claros signos de violencia causada por arma blanca. Son seis los casos identificados, todos hombres, de los que tan solo uno sobrevivió (sepultura 78) (Tabla 2).

\begin{tabular}{|c|c|c|c|c|}
\hline Sepultura & Edad & Sexo & HG SNRY & HG mt \\
\hline 32 & Adulta & Hombre & R1 & H1 \\
\hline 34 & Adulta joven & Hombre & F & U \\
\hline 61 & Juvenil & Hombre & F & U6a \\
\hline 78 & Adulta & Hombre & E1b1b1b & H1 \\
\hline 92 & Adulta joven & Hombre & & HVO $^{*}$ \\
\hline 93 & Juvenil & Hombre & & U5 \\
\hline
\end{tabular}

Tabla 2. Identificación genética de los hombres con lesiones por arma blanca

(De Miguel et ál., 2016, 175, tabla 9.4).

De los seis casos se identificó el ADN del cromosoma Y en cuatro de ellos, de los que tres son más frecuentes en Europa (sepulturas 32, 34, 61), lo que hace considerar que sus padres no eran de origen africano. De estos tres casos, dos tienen marcadores maternos africanos (sepulturas 32 y 61), entre los que destaca el individuo 61, ya que su haplogrupo mitocondrial U6a está claramente documentado entre los bereberes del Noroeste africano, alcanzando el 80\% en algunas regiones de Marruecos (Fontecha, 2013: 321). En el hombre adulto de la sepultura 34, ambos marcadores genéticos son considerados europeos. En los casos en los que solo se ha podido determinar el ADN mitocondrial, por lo que solo conocemos la vía materna, uno indica un origen africano (sepultura 92) y el otro (sepultura 93) un origen claramente local.

Estos casos de violencia bien testimoniada en la maqbara de Pamplona, y presente en otros cementerios islámicos peninsulares como Baza (Ríos y Pérez, 2008), Murcia (Pimentel et ál., 2001) y Tudela (Bienes, 2007) durante el predominio islámico, nos ilustran sobre un momento de claro conflicto, si bien no hay datos por el momento de las circunstancias en las que se desarrollan los mismos. Actualmente, no se conocen en tierras navarras fosas de batallas, como ocurre en Alarcos (Muñoz y Herrerín, 2016). En Pamplona se han localizado restos con evidencias de muerte violenta dispersos en el área cementerial de la maqbara, al igual que ocurre en otros casos conocidos en Tudela (calle Herrerías), en contexto musulmán, y en Arizkoa (Monreal) (De Miguel, 2020), en contexto cristiano.

Un aspecto vinculado con la asignación sexual de los esqueletos que muestran una muerte violenta, o evidencias de traumas de origen en episodios de violencia interpersonal, es que todos los conocidos actualmente son hombres. La edad varía, habiéndose documentado en Pamplona dos casos en juveniles (15-20 ańos), hasta la edad adulta madura (Tabla 2). Este dato nos indica, muy probablemente, la asunción temprana de actividades relacionadas con el manejo de las armas, dejando a las mujeres alejadas de 
esta actividad. No obstante, hay testimonios como los de la maqbara de Baza, en la que también hay mujeres con signos de muerte violenta, si bien a partir de la localización de las lesiones parecen corresponder con un episodio de acoso y huida (Ríos y Pérez, 2008).

Entre los aspectos conocidos por la tesis doctoral de Lara Fontecha (2013) hay uno que destaca por su relevancia, el reconocimiento de marcadores genéticos claramente africanos en un porcentaje considerable de la población exhumada en Pamplona. Es significativamente más elevado el número de individuos con marcadores africanos entre los hombres, llegando a ser una tercera parte de los estudiados. Entre las mujeres también se constata la representación genética africana. Este estudio permite reconocer de forma fiable la presencia de población alóctona tanto en el grupo de los hombres, de forma mayoritaria, como en el de las mujeres, en este caso de manera más reducida (Fontecha, 2013; De Miguel et ál., 2016). Es cierto que los resultados no siempre son claros con respecto al origen de las poblaciones ya que los genes están repartidos tanto por África, como por el territorio peninsular y Europa, de modo que tener la seguridad de un origen concreto es sumamente complicado.

Hemos estudiado otra cuestión importante, si bien de forma muy parcial por el momento: los isótopos de movilidad que permiten inferir si las personas estudiadas nacieron o no en el mismo lugar en el que fueron enterradas. De los dos casos analizados de nuestra población, dos mujeres (sepulturas 28 y 131), una de ellas, la sepultura 131, tiene marcadores genéticos preferentemente localizados en el norte de África; en el caso de la sepultura 28, los marcadores están presentes a ambos lados del Mediterráneo no siendo determinante los resultados genéticos para determinar su origen geográfico. Los isótopos indicaron un origen ajeno a las tierras pamplonesas, proponiendo en el estudio un más que posible origen norteafricano (Prevedorou et ál., 2010).

Un aspecto que resultó novedoso es el reconocimiento de modificaciones dentales de carácter intencional. Estas denticiones se han estudiado a través de microscopía electrónica de barrido (EMB), confirmando que no son desgastes de origen alimentario ni por el uso de los dientes en actividades de tipo artesanal o laboral. Hay que relacionarlas con una intencionalidad estética, o como marca de pertenencia a un grupo, probablemente relacionado con ritos de paso (Romero et ál., 2009). Queremos reseñar la existencia de un hadìt, recogido entre otros por Abi Al-Husayn, XXV (5301), que prohíbe explícitamente a las mujeres el limado de los dientes, parece que vinculado con la intención de las mismas de parecer más jóvenes presentando los dientes separados: (5301) 'Abdullah dijo: "Allah maldijo a las mujeres que tatúan y a las que se hacen tatuar, a las que se depilan la cara y a las que se hacen depilar y a las que se separan (acortan) los dientes para embellecerse cambiando lo que Allah ha creado». Esta referencia permite sugerir que probablemente en parte del territorio islamizado se realizaba de forma habitual este tipo de modificaciones corporales, lo que llevo a indicar su prohibición.

De las denticiones que tienen modificaciones culturales en los dientes, algunas de ellas tienen marcadores genéticos africanos (Tabla 3). No obstante, nada es simple, y mucho menos cuando hablamos de relaciones humanas. Al menos en un caso de las mujeres que muestran manipulaciones dentales de origen intencional su genética mitocondrial es claramente navarra, lo que nos indica una historia familiar por vía materna de mujeres 
plenamente asentadas en el solar navarro. En este caso desconocemos el origen genético paterno que bien pudiera ser africano, como ya hemos indicado ante la casi segura mezcla entre hombres recién llegados con mujeres locales.

A través de los datos genéticos podemos conocer el origen de las mujeres y hombres que presentaban manipulaciones dentales de origen cultural. Dos de los tres hombres muestran haplogrupos en el cromosoma Y claramente africanos (sepulturas 13 y 107), siendo en el caso del 107 de origen bereber; además, los datos del ADNmt también indican un origen africano, como ocurre con el 13, en el que igualmente ambos progenitores fueron de origen genético africano. En el grupo de las mujeres, cuatro poseen marcadores genético mitocondriales africanos (sepulturas 85, 131, 143 y 145); en el caso de las mujeres tan solo podemos determinar, por el momento, la genética materna. Era de esperar que todas las mujeres y hombres con manipulaciones dentarias de origen cultural fueran genéticamente africanas; sin embargo, es de gran relevancia el hecho de que seis de las personas identificadas muestren genes europeos, destacando la mujer de la sepultura 194 cuyos genes son totalmente locales (U5).

\begin{tabular}{|c|c|c|c|c|}
\hline Sepultura & Edad & Sexo & HG SNRY & HG mt \\
\hline 13 & Adulta & Hombre & E & HVO $^{*}$ \\
\hline 34 & Adulta joven & Hombre & F & U \\
\hline 85 & Adulta & Mujer & & L2b \\
\hline 86 & Adulta & Mujer & & J2b \\
\hline 107 & Adulta madura & Hombre & E1b1b1b & H5 \\
\hline 120 & Juvenil & Mujer & & K1a* \\
\hline 131 & Adulta joven & Mujer & & H3 \\
\hline 143 & Adulta joven & Mujer & & L3f1b4a \\
\hline 145 & Adulta & Mujer & & H \\
\hline 159 & Adulta & Mujer & & HVO* \\
\hline 193 & Juvenil & Mujer & & J2 \\
\hline 194 & AM & Mujer & & U5 \\
\hline
\end{tabular}

TABLA 3. Manipulaciones dentarias intencionales y resultados genéticos

(De Miguel et ál., 2016: 175, tabla 9.4).

\section{Lo QUe nos cuenta la población islámica de Bambeluna (Pamplona)}

Si algo han aportado los estudios realizados de los hallazgos en la maqbara de Pamplona, es el descubrimiento inesperado de una población, cuyo testimonio, silenciado casi totalmente por las fuentes escritas, se convierte en incontestable a la luz de su presencia.

El ritual se convierte en el mejor testimonio cultural posible ante la carencia de otros materiales que permitan aclarar su adscripción cultural. 
Las investigaciones realizadas sobre la posible presencia islámica en Pamplona a inicios del siglo viII se basan en unas fuentes escritas muy posteriores a la época de los hechos, en las que se hacía referencia a cinco hitos importantes recogidos en un trabajo previo (De Miguel, 2016).

El primero, la existencia del pacto de Pamplona firmado alrededor de 714-718, considerado similar al de Tudmir, en el que se fijaban las normas y relaciones entre el nuevo poder islámico y la autoridad local, con cierto margen de respeto ante la convivencia de las dos religiones, vinculadas a diferentes elites y poderes. El segundo es el que nos indica que las tropas musulmanas llegaron hasta Poitiers (732), utilizando la vía que pasaba por Pamplona para llegar al paso fronterizo que les introducía en tierras francas. Se infiere que la ciudad era un lugar propicio para el ejército musulmán, probablemente al ejercer el control político y militar sobre Pamplona y su territorio, bien de forma hegemónica o en forma de pacto con las elites cristianas. El tercero son las referencias a los disturbios acaecidos entre la población islámica recientemente llegada y su poder central, resuelto por el valí 'Uqba (734) a quien se le atribuye la "pacificación», o represalia según se mire, y el aporte de población musulmana a los nuevos territorios conquistados y pacificados, entre ellos la Pamplona islámica o Bambeluna. Disponemos del texto de Ibn 'Idārī alMarrākušì que dice: «Fue costumbre de este Ocba combatir á los idólatras todos los años, y les tomaba sus ciudades siendo el que conquistó la ciudad de Arbona, y sometió la Galiquia y Bambeluna, que hizo poblar de muslimes llegando a comprender sus conquistas todo el territorio de Galiquia» (1860: 71, trad. F. Fernández). El cuarto acontecimiento es la batalla de Roncesvalles, relacionada con la destrucción de las murallas de Pamplona durante el regreso de Carlomagno de su fallida conquista de Zaragoza. Es en la Canción de Roldán donde se recogen numerosas referencias a la presencia de «sarracenos», incluyendo muy posiblemente a la población islámica asentada en Bambeluna, mentada en la Crónica Silense como oppidum Maurorum: Porro cum in reditu Pampiloniam Maurorum oppidum destruere conaretur, pars máxima exercitus sui | in [5] ipso Pireneo iugo magnas exsolvit penas (Santos Coco, 1921: 17). El quinto hito sería el asesinato de Mutarrif ibn Musa, un personaje con la suficiente relevancia social, y quizás política, para haber dejado constancia de su asesinato, que se asocia con el final del control islámico de la ciudad y de su territorio.

Si estos datos eran los mejor conocidos durante la historia del siglo vin de la ciudad de Pamplona, casi quedaron en el olvido ante la inexistencia de evidencias materiales que permitieran afianzar el conocimiento sobre el posible control musulmán del solar pamplonés (Faro et ál., 2007-2008).

Sabido esto, la maqbara se convierte en el testimonio material más evidente de la presencia de población islámica y/o islamizada durante el siglo viII. Y son los esqueletos recuperados, y generalmente bien conservados, los que nos cuentan las historias vividas por personas hasta ahora anónimas y que, de alguna manera, resucitan permitiendo reconstruir, aunque sea parcialmente, una parte de nuestra propia historia.

De los datos demográficos podemos proponer que estamos ante una población normal para una comunidad histórica prevacunal, formada casi a partes iguales entre la población infantil-subadulta y la adulta (Gráfico 1). 
Un aspecto que diferencia de forma evidente a los hombres de las mujeres en la población son las muertes de causa violenta, documentadas en cinco hombres, a lo que debemos sumar fracturas y traumatismos claramente vinculados con enfrentamientos interpersonales, en estos casos con signos claros de supervivencia. Estos hechos nos permiten inferir, a partir de los datos disponibles, que las mujeres no tuvieron participación en episodios violentos y que nada indica que dentro de sus funciones estuvieran las relacionadas con el uso de armas, que quedaría de forma exclusiva como competencia de los hombres.

En relación con los datos genéticos disponibles a partir de la tesis doctoral de Lara Fontecha (2013), ya hemos mencionado la mayor representación de hombres con genes norteafricanos en el cromosoma $\mathrm{Y}$, siendo dos tercios de los casos estudiados. Para el grupo de las mujeres es menor el porcentaje de ADN mitocondrial de origen claramente africano, si bien no descartamos que parte de las mujeres, cuyos marcadores genéticos son poco precisos entre el norte de África y el sur de Europa, fueran originarias del Magreb como parece confirmar el estudio isotópico realizado de las sepulturas 28 y 131 .

Si enlazamos los datos genéticos, los isótopos estables y las modificaciones dentales de carácter cultural, con origen claramente africano, se nos abre un panorama interpretativo sumamente interesante. En primer lugar el hecho de que la mayor parte de las modificaciones dentales se den en mujeres (9/3), cuando los hombres están numéricamente mejor representados en el conjunto poblacional (Tabla 4). A partir de la presencia de una mujer con marcadores genéticos maternos locales y manipulaciones dentales de origen cultural africano, podemos considerar este caso como una evidencia clara de aculturación de al menos parte de la población. Si consideramos que aculturación es un proceso por el que se adquieren tradiciones culturales ajenas a la propia, mostrando rasgos identificables. En nuestro caso las manipulaciones dentarias como rasgo visible de pertenencia a un grupo claramente diferente de las personas cristianas, que no muestran esas manipulaciones. A lo que sumamos algo más profundo y menos permeable a los cambios, el rito funerario, probablemente como clara muestra de su conversión al Islam, como miembros de pleno derecho y con las mismas obligaciones que la población musulmana llegada desde África.

Si inicialmente supusimos que las marcas dentales pudieron estar ya realizadas cuando llegó el grupo de población islámica a la ciudad, a la luz de los resultados debemos considerar que, al menos en algunos casos, la práctica del tallado y limado de los dientes se realizó en Bambeluna (Pamplona), quizás como un rito de paso entre la infancia y la edad adulta, dado que dos mujeres ya las presentan a la edad juvenil cuando fallecieron. Lo que nos llama la atención es que, si la práctica se realizó en la ciudad de acogida, sean relativamente tan pocos los casos que hemos documentado. La propuesta de que fueran prácticas reducidas a una familia o grupo del mismo origen local no parece poder justificarse, dado que los datos obtenidos (Fontecha, 2013; De Miguel et ál., 2016) sobre relaciones de parentesco entre personas de la maqbara han dado como resultado la agrupación de personas con manipulaciones dentales con otros enterramientos que no las presentaban, no identificándose en ninguno de los grupos familiares documentados más de una persona con manipulaciones dentales culturales. 
Si unimos los datos culturales con las aportaciones de la genética, observamos la complejidad de la comunidad estudiada, en la que se reconocen personas, tanto hombres como mujeres, de origen africano junto con otras de origen local, y en el caso de algunos hombres de los que conocemos tanto la genética paterna como la materna, sus genes son tanto alóctonos como locales. Por lo tanto, hay representados individuos en los que los genes paternos y los maternos son africanos, en otros ambos locales, y en otros, para evidenciar la complejidad, son los padres alóctonos y las madres locales, mientras que en otras personas lo son a la inversa.

Consideramos que con los datos ofrecidos hacemos visibles evidencias que nos permiten sustentar las propuestas de aculturación de parte de la población local, a la vez que podemos identificar, en varios de los casos, quiénes pertenecían a la población local y quiénes llegaron desde uno o varios puntos aun imprecisos de África, siguiendo el progreso del Islam desde Oriente hacia Occidente por todo el norte africano. A la vez, podemos justificar que hubo una vinculación genética entre la población recién llegada y la residente en la ciudad.

La potencialidad de este tipo de estudios, en los que se combinan los datos históricos, el registro arqueológico, los estudios osteoarqueológicos, los análisis isotópicos y la genética, nos ofrece una visión de una realidad histórica lejana en el tiempo, narrada a través de sus protagonistas. Son las historias contadas por los huesos, parcialmente impresas en lo que queda de sus cuerpos, pero difícilmente engańosas.

\section{A MODO DE CONCLUSIÓN}

La maqbara de Pamplona-Bambeluna es a día de hoy la más antigua identificada en la península Ibérica, ciñéndose su uso al siglo viII, sin perduración posterior documentada.

Demográficamente muestra una población con una mortalidad propia de las sociedades prevacunales, con una elevada representación de población infantil y juvenil que no ha alcanzado la edad adulta.

Aunque hay una mayor representación de hombres que de mujeres, sabiendo que la excavación es parcial, consideramos que es una representación casi equilibrada.

Las evidencias patológicas son muy variadas, lo que nos informa de una sociedad donde la enfermedad y el apoyo del grupo ante la adversidad están claramente integrados en su imaginario como parte de la cotidianeidad.

Las muertes violentas en cinco casos, además de otros en los que se identifican traumatismos producidos por enfrentamientos interpersonales, se observan solo en hombres, lo que muestra una sociedad habituada a la violencia, con al menos parte del grupo de hombres, si no todos, que ejerció funciones militares que excluyeron claramente a las mujeres.

El estudio genético disponible muestra una alta complejidad poblacional, en la que dos tercios de los hombres estudiados tienen marcadores paternos africanos, siendo menor en el caso de las mujeres, si bien en ellas solo se conocen los marcadores genéticos 
maternos. En la población hay hombres y mujeres con genética africana, local y mixta. Tenemos por tanto representada la primera generación de población africana y las posteriores combinadas con gentes locales conversas.

Las manipulaciones dentales de origen cultural se realizaron preferentemente entre las mujeres, aunque no fueron excluyentes para con los hombres. Quienes las portan son tanto de origen africano, como local y mixto, por lo que probablemente en algunos casos las modificaciones se hicieron en la ciudad.

Estos datos permiten reconocer la complejidad genética presente en una población pequeña, informándonos de manera clara de la mezcla de poblaciones, cuyo nexo de unión se fundamenta en la religión. Este hecho permite inferir que parte de la población se convirtió al Islam, no solo mujeres como habíamos propuesto al inicio de la investigación, sino también hombres, creándose una comunidad mixta en origen y unificada por las creencias religiosas, y quizás por el atractivo que genera la proximidad a los ámbitos de poder.

\section{Agradecimientos}

Queremos agradecer expresamente la generosidad de la Dra. Lara Fontecha quien nos ha facilitado el acceso a los datos inéditos de su tesis doctoral, al igual que a las directoras de la misma las Dras. Concepción de la Rúa y Neskuts Izagirre. A mi directora y director de tesis, la Dra. Sonia Gutiérrez Lloret y el Dr. Mauro S. Hernández Pérez, de la Universidad de Alicante. Igualmente, a la Dra. Olatz Villanueva Zubizarreta por la oportunidad de participar en esta publicación, haciendo visible una población de tan alto interés como es la representada en la maqbara de Pamplona. A Mercedes Unzu, Fernando de Miguel Induráin y Ricardo de Miguel Ibáñez por su apoyo y colaboración.

\section{REFERENCIAS BIBLIOGRÁFICAS}

Abi Al-Husayn Muslim Ibn Al-Hâŷyâŷy Al-Qushayrī Al-Naysabūrī, 2 (trad. al castellano de 'Abdu Rahman Colombo al-Ŷerrāhī). Oficina de Cultura y Difusión Islámica Argentina: 12431245. http://d1.islamhouse.com/data/es/ih_books/parts/Sahih_Muslim/es_02_Los_funerales_en_el_Islam.pdf (acceso 1/10/2019)

Almagro Basch, Martín. La necrópolis hispano-visigoda de Segóbriga, Saelices (Cuenca). Madrid: Ministerio de Educación y Ciencia, 1975. Excavaciones Arqueológicas en España, 84.

Balthazard, V. y Dervieux, H. «Études anthropologiques sur foetus humain». Annales de Médicine Légale, 1921, vol. 1, pp. 37-42.

Bienes Calvo, Juan José. «La necrópolis islámica de la calle Herrerías (Tudela)». En La tierra te sea leve. Arqueología de la muerte en Navarra. Pamplona: Gobierno de Navarra. Departamento de Cultura y Turismo-Institución Príncipe de Viana, 2007, pp. 253-258.

Bocquet-Appel, Jean Pierre. La paléodémographie, 99'9 \% de l'histoire démographique des hommes ou la démographie de la Préhistoire. Paris: Editions Errance, 2008.

Buikstra, Jane Ellen y Ubelaker, Douglas H. Standards for Data Collection from Human Skeletal Remains. Fayetteville: Arkansas Archeological Survey, 1994. Research Series, 44. 
Corán (ed. preparada por Julio Cortés). 8. ${ }^{\mathrm{a}}$ ed. Barcelona: Herder, 2002.

De Miguel Ibáñez, María Paz. «Anexo: Estudio osteoarqueológico». En Trelis Martí, Julio; Ortega Pérez, José Ramón; Reina Gómez, Inmaculada y Esquembre Bebiá, Marco Aurelio. «El cementerio mudéjar del Raval (Crevillent-Alicante)», Arqueología y Territorio Medieval, 2009, vol. 16, p. 210-216.

De Miguel Ibáñez, María Paz. La maqbara de Pamplona (s. VIII). Aportes de la osteoarqueología al conocimiento de la islamización en la Marca Superior. Alicante: Biblioteca Virtual Miguel de Cervantes, 2016. http://www.cervantesvirtual.com/nd/ark:/59851/bmc8d1w3

De Miguel Ibáñez, María Paz. «Ficha 33, lesión por arma blanca, en mandíbula y tercera cervical. Posible decapitación». En La vida impresa en los huesos. Paleopatología en Navarra. Catálogo de la exposición. Pamplona: Museo de Navarra-Gobierno de Navarra, 2020.

De Miguel Ibáñez, María Paz; Fontecha Martínez, Lara; Izagirre Arribalzaga, Neskuts y De la RÚa VACA, Concepción. «Paleopatología, ADN y diferenciación social en la maqbara de Pamplona: límites y posibilidades». En Quirós Castillo, José Antonio (ed.). Demografía, paleopatologias y desigualdad social en el norte de la Península Ibérica en época medieval (coloquio). Bilbao: Universidad del País Vasco, 2016, pp. 163-182.

Duday, Henri; Courtaud, Patrice; Crubezy, Eric; Sellier, Pascal y Tillier, Anne-Marie. "L’Anthropologie "de terrain": reconnaissance et interprétation des gestes funéraires». Bulletins et Mémoires de la Société d'Anthropologie de Paris, 1990, nouvelle série, vol. 2, n. ${ }^{\circ} 3-4$, pp. 29-50.

Etxeberria, Francisco; Baraybar, José Pablo; De la Rúa Vaca, Concepción y Vegas Aramburu, José Ignacio. «Identificación postmortem y mecanismos de producción de las fracturas diafisarias de cúbito». En Villalaín Blanco, José Delfín; Gómez Bellard, Carlos y Gómez Bellard, Francisco (coords.). Actas del II ${ }^{\circ}$ Congreso Nacional de Paleopatología. Valencia: Ministerio de Educación y Ciencia-Universitat de València, 1996, pp. 319-323.

Faro Carballa, José Antonio; García-Barberena Unzu, María y Unzu Urmeneta, Mercedes. «Pamplona y el Islam. Nuevos testimonios arqueológicos». Trabajos de Arqueología Navarra, 2007-2008, vol. 20, pp. 229-284.

FazeKas, István Gyula y Kósa, F. Forensic Fetal Osteology. Budapest: Academiai Kiadò, 1978.

Ferembach, Denise; Schwidetzky, I. y Stloukal, Milan. «Recommandations pour déterminer l'âge et le sexe sur le squelette». Bulletins et Mémoires de la Société d'Anthropologie de Paris, 1979, série XIII, vol. 6, n. ${ }^{\circ}$ 1, pp. 7-45.

Fontecha Martínez, Lara. Análisis genético de la maqbara de Pamplona (Navarra, s. VIII) una ventana a la invasión islámica en el norte de la peninsula Ibérica. Departamento de Genética, Antropología Física y Fisiología Animal. Universidad del País Vasco/Euskal Herrico Unibersitatea, 2013. Tesis doctoral. Inédita.

Galvé Izquierdo, María Pilar. «Necrópolis islámica de la Puerta de Toledo (Zaragoza): Nuevas excavaciones». En Torres Palomo, María Paz y Acién Almansa, Manuel (eds.). Estudios sobre cementerios islámicos andalusies. Málaga: Universidad de Málaga, 1995, pp. 117-136. Estudios y Ensayos.

García-Barberena Unzu, María. El urbanismo de la ciudad de Pamplona entre los siglos VII a. C. y VIII d. C. Universidad de Navarra, 2016. Tesis doctoral. Inédita.

Gutiérrez Lloret, Sonia. «De Teodomiro a Tudmīr. Los primeros tiempos desde la arqueología (s. VII-IX)». En De Mahoma a Carlomagno: los primeros tiempos (siglos VII-IX). XXXIX Semana de Estudios Medievales, Estella, 17-20 de julio de 2012. Pamplona: Gobierno de Navarra. Departamento de Cultura, Turismo y Relaciones Institucionales, 2013, pp. 229-283. 
Ibn Idari Al-Marrakusi. Historias de al-Andalus (trad. de F. Fernández González). Granada: Imp. de D. Francisco Ventura y Sabatel, 1860. http://goo.gl/QN8NDl. http://www. bibliotecavirtualdeandalucia.es/catalogo/consulta/resultados_navegacion.cmd?busq_ autoridadesbib=BAA20070016978 (Acceso 21/8/2015)

Jeanty, Philippe; Rodesch, Frederic; Delbeke, Dominique y Dumont, Jacques E. «Estimation of Gestational Age from Measurements of Fetal Long Bones». Journal Ultrasound in Medicine, 1984, vol. 3, n. ${ }^{\circ}$ 2, pp. 75-79.

Muñoz Ugarte, Lucía y Herrerín, Jesús. "Un ejemplo de violencia procedente de la Batalla de Alarcos (1195)». En Asociación Española de Antropología y Odontología Forense. VIII Reunión Cientifica. La Granja. Segovia, 2016, pp. 49-50.

Nunes de Mendonça, Maria Cristina. Contribución para la identificación humana a partir del estudio de las estructuras óseas. Determinación de la talla a través de la longitud de los huesos largos. Universidad Complutense de Madrid. Facultad de Medicina, 1998. Tesis doctoral. biblioteca.ucm.es/tesis/19972000/D/0/D0119101.pdf

Pearson, Karl. «IV. Mathematical contributions to the theory of evolution. V. On the reconstruction of the stature of prehistoric races». Philosophical Transactions of the Royal Society of London, 1899, Series A, vol. 192, pp. 169-244.

Pimentel de Francisco, G.; Rodríguez González, A. I.; González Martín, Armando; Campo Martín, Manuel; Robles Rodríguez, Francisco José y Arzoz del VAL, L. «Evidencias de violencia en la población hispanomusulmana de San Nicolás (Murcia, s. XI a s. XIII)». En Campo Martín, Manuel y Robles Rodríguez, Francisco José (eds.). ¿Dónde estamos? Pasado, presente y futuro de la Paleopatología. Actas del VI Congreso Nacional de Paleopatología. Madrid: Universidad Autónoma de Madrid-Asociación Española de Paleopatología, 2001, pp. 542-544.

Prada Marcos, María Encina. Estudio antropológico de los restos humanos medievales y modernos de la necrópolis leonesa de Palat del Rey. Universidad de León. Facultad de Biología. Dpto. de Biología Animal. Antropología, 1993. Tesis doctoral. Inédita.

Prevedorou, E.; Díaz-Zorita Bonilla, M.; Romero, A.; Buikstra, J. E.; De Miguel Ibáñez, M. P. y Knudson, K. J. «Residential Mobility and Dental Decoration in Early Medieval Spain: Results from the Eighth Century Site of Plaza del Castillo, Pamplona». Dental Anthropology, 2010, vol. 23, n. ${ }^{\circ}$ 2, pp. 42-52.

Ríos Frutos, Luis y Pérez Asensio, Manuel. "Trauma peri mortem en la maqbara medieval de Baza, Granada». En Roca de Togores Muñoz, Consuelo y Rodes Lloret, Fernando (eds.). Actas de las Jornadas de Antropología Física y Forense. Alicante: Instituto Alicantino de Cultura Juan Gil-Albert, 2008, pp. 87-99.

Roca DE Togores, Consuelo. «Estudio antropológico y paleopatológico». En La maqbara del Tossal de Manises (Alicante). Tomo II. Alicante: Museo Arqueológico de Alicante-Diputación de Alicante, 2008, pp. 9-70. Serie Excavaciones Arqueológicas. Memorias, 4.

Romero Rameta, Alejandro; Buikstra, Jane E.; De Miguel Ibáñez, María Paz; Knudson, K. J.; Prevedorou, E. A.; Díaz-Zorita Bonilla, M. y De Juan, J. «Mutilación dentaria en la necrópolis islámica de Plaza del Castillo (siglo viII d. C.) de Pamplona (Navarra)». Revista Española de Antropología Física, 2009, vol. 29, pp. 1-14.

Santos Coco, Francisco. Historia Silense. Madrid: Centro de Estudios Históricos, 1921. http:// bibliotecadigital.jcyl.es/i18n/catalogo_imagenes/grupo.cmd?path=10066520

Scheuer, Louise y Black, Sue. Developmental Juvenile Osteology. Amsterdam: Elsevier Academic Press, 2000. 
Schutкоwsкi, Holger. «Sex determination of infant and juvenile skeletons: I. Morphognostic features». American Journal of Physical Anthropology, 1993, vol. 90, n. o 2, pp. 199-205.

Serrano, José Luis y Castillo, Juan Carlos. «Las necrópolis de Marroquíes Bajos (Jaén). Avance de las investigaciones arqueológicas». Arqueología y Territorio Medieval, 2000, vol. 7, pp. 93-120.

Tendero Porras, Eva; Guilabert Mas, Antonio P. y Olcina Doménech, Manuel H. La maqbara del Tossal de Manises (Alicante). Tomo I: Estudio Arqueológico. Alicante: Museo Arqueológico de Alicante-Diputación de Alicante, 2007. Serie Excavaciones Arqueológicas. Memorias, 4.

Ubelaker, Douglas H. Enterramientos humanos: excavación, análisis, interpretación. Donosti-San Sebastián: Sociedad de Ciencias Aranzadi, 2007. Munibe suplemento 24. Gehigarria.

Vigil-Escalera Guirado, Alfonso. «Sepulturas, huertos y radiocarbono (siglos viII-XIII d. C.). El proceso de la islamización en el medio rural del centro peninsular y otras cuestiones». Studia Historica. Historia Medieval, 2009, vol. 27, pp. 97-118. 